\title{
Research on Operation Optimization Technology of Integrated Energy System Based on Multiple Subjects
}

\author{
Xiaojing Dong ${ }^{1}$, Wang Su${ }^{1}$, Hankui Tian², Zelong Zhang ${ }^{1}, \mathrm{Xu} \mathrm{Han}^{2,{ }^{*}}$ and Mengyuan Tang ${ }^{1}$ \\ ${ }^{1}$ Economic and Technical Research Institute of State Grid Ningxia Electric Power Co., Ltd. China \\ ${ }^{2}$ North China Electric Power University, China
}

\begin{abstract}
With the development of social economy and technology, the coupling relationship between different energy supply systems (cold, heat, electricity, gas, etc.) has become closer, and a comprehensive energy system with multiple subjects has become a development trend. In order to pursue a clean and efficient energy system, increase the consumption rate of new energy and reduce operating costs, a multiagent integrated energy system operation optimization method including combined heat and power (CHP) operators and photovoltaic user groups is proposed. In this paper, the distributed operation optimization method of integrated energy system based on multiple agents is studied. Considering the demand response of electric energy and thermal energy, and considering economy and user satisfaction, the distributed optimization scheduling model of integrated energy system with multiple agents is established to achieve the centralized optimization effect.
\end{abstract}

\section{Introduction}

Efficient, clean, and low-carbon are the mainstream directions of energy development in the world today. The development of a comprehensive energy system that realizes the deep integration of new technologies in the fields of energy and information, and adapts to the development of distributed energy, diversified (cold, heat, electricity, gas, etc.) energy demand and other new business forms, has become an objective requirement and inevitable choice of the energy revolution [1]. Compared with traditional energy production, the combined heat and power unit [2,3] can simultaneously supply electricity and heat to users, which greatly improves the operating efficiency of the system. In addition, due to the advantages of cleanliness and high efficiency, distributed photovoltaic [4,5] (photovoltaic, PV) systems have developed rapidly. For the above reasons, integrated energy systems including CHP systems and photovoltaic user groups are increasing. In order to improve the consumption rate of renewable energy, the optimal operation of integrated energy systems (IES) with multiple entities has become an urgent problem to be solved.

Based on this, this paper proposes a distributed operation optimization method for a multi-agent integrated energy system. By establishing a demand response model that takes into account electrical and thermal energy, energy sharing between users and energy sharing between CHP operators and users are established. The basic optimization model formed by CHP operators and photovoltaic user groups can minimize system operating costs and improve user satisfaction.

\section{Energy sharing architecture of integrated energy system with multi- agent}

The research in this paper focuses on the integrated energy system with CHP operators and several photovoltaic users. The cogeneration operator is the operator of the integrated energy system. It manages the cogeneration micro-combustion unit and thermal energy storage, and can provide electricity and heat to the photovoltaic users in the system. The configuration of thermal energy storage on the source side breaks the rigid constraint of "using heat to determine electricity", which can effectively improve the regulation capability and system flexibility of the micro-gas turbine [6]. The operator's CHP is equipped with a combined heat and power energy management system (CHP-EMS), in which the CHP completes optimal scheduling, and can determine the CHP's power generation, heat production, and heat storage and release of thermal energy storage. Thermal power. As shown in Figure 1 below, it is a schematic diagram of the interaction mechanism of the integrated energy system with CHP, which includes: the main body of integrated energy service, also called energy hub operator (EHO), and some user clusters (USER) containing photovoltaics As well as energy storage operators (ESO), cogeneration units, users with photovoltaic power generation devices (PV), users without photovoltaic power generation devices (no-PV),

\footnotetext{
*Corresponding author: hanxuyy412@163.com
} 
and heat storage equipment, HSE) and battery storage equipment (BSE).

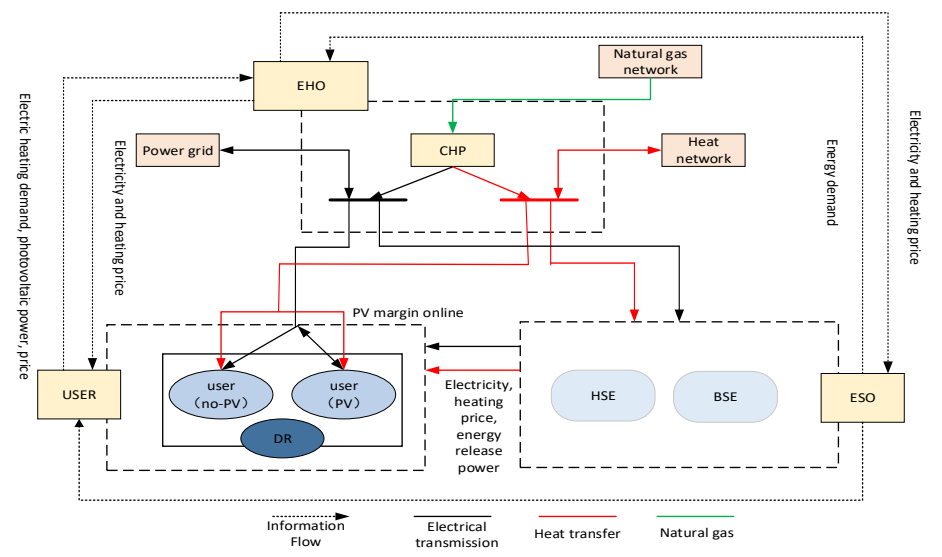

Fig. 1. The interaction mechanism of the integrated energy system with CHP

\section{System model}

\subsection{CHP operator model}

\subsubsection{CHP system}

The core device of the cogeneration system is a micro turbine generator (MTG), and the relationship between the fuel cost and the output of the unit is [7]:

$$
C_{c h p}=P_{g a s} \frac{P_{c h p} \Delta t}{\eta_{c h p} L_{H V N G}}
$$

Where: $C_{c h p}$ is the fuel cost of the micro-gas turbine; $P_{c h p}$ is the electric power of the micro-gas turbine; $P_{g a s}$ is the price of natural gas; $\eta_{c h p}$ is the power generation efficiency of the micro-gas turbine; $\Delta t$ is the length of the time period; $L_{H V N G}$ is the low heating value of natural gas.

The thermoelectric relationship model of the cogeneration system is:

$$
H_{c h p}=\frac{\left(1-\eta_{c h p}-\eta_{L}\right) \eta_{h} C_{o p h} P_{c h p}}{\eta_{c h p}}
$$

Where: $H_{c h p}$ is the heating power of the micro-gas turbine; $\eta_{L}$ is the heat loss rate; $C_{o p h}$ and $\eta_{h}$ are the heating coefficient and flue gas recovery rate of the bromine cooler, respectively.

\subsubsection{Thermal energy storage}

Due to the mismatch between the size of the thermoelectric load and the peak-valley period, CHP will be subject to the rigid constraints of "heating by heat" or "heating by electricity" and cannot operate efficiently and economically. Thermal energy storage can alleviate the contradiction of electric and heating load mismatch, and on the basis of meeting the electric and thermal energy demand in the microgrid, coordinate CHP to realize unified electric and heating dispatching [8].
The heat storage and release of thermal energy storage usually cause heat loss, which is described in the form of the heat storage state equation as:

$$
Q_{t+1}^{t e s}=e^{-\gamma \Delta t} Q_{t}^{t e s}+\left(H_{t}^{i n} \eta_{i n}-\frac{H_{t}^{d r}}{\eta_{d r}}\right) \Delta t
$$

Where: $\gamma$ is the attenuation coefficient; $Q_{t+1}^{t e s}$ is the capacity of thermal energy storage at time $t ; H_{t}^{i n}$ and $H_{t}^{d r}$ are the heat storage and heat release power of thermal energy storage at time $t ; \eta_{i n}$ and $\eta_{d r}$ are the heat storage and heat release efficiency, respectively.

Since the attenuation coefficient is usually very small, in order to facilitate the calculation, linearize (3) to obtain (4):

$$
Q_{t+1}^{t e s}=(1-\gamma \Delta t) Q_{t}^{t e s}+\left(H_{t}^{i n} \eta_{i n}-\frac{H_{t}^{d r}}{\eta_{d r}}\right) \Delta t
$$

The charging and discharging heat power of thermal energy storage should meet constraint formula (5); the upper and lower limits of the capacity of thermal energy storage should meet constraint formula (6).

$$
\begin{gathered}
0 \leq H_{t}^{i n} \leq \frac{1}{\eta_{\text {in }}} H_{\text {in }}^{\max } 0 \leq H_{t}^{d r} \leq H_{\max }^{d r} \eta_{d r} \\
Q_{\min }^{\text {tes }} \leq Q_{t}^{\text {tes }} \leq Q_{\max }^{\text {tes }}
\end{gathered}
$$

Where: $H_{i n}^{\max }$ is the maximum value of thermal energy storage heat storage power; $H_{\max }^{d r}$ is the maximum value of thermal energy storage heat release power; $Q_{\min }^{t e s}$ and $Q_{\max }^{t e s}$ are the upper and lower limits of thermal energy storage capacity, respectively.

\subsubsection{CHP operating costs}

CHP operators supply electricity and heat to users, and connect excess electricity to the Internet. The cost mainly includes 4 parts: 1) the cost of power generation of CHP; 2) the income of selling electricity to the large grid; 3) the income of selling heat to users; 4) the income of selling electricity to users. The operator's cost $C_{\text {ope }}$ is as follows: 


$$
\begin{aligned}
\min C_{\text {ope }} & =C_{c h p}\left(P_{c h p}, H_{c h p}\right)-P_{s}\left(P_{c h p}-P_{p r o}\right) \\
& -\gamma H_{p r o}-\lambda P_{p r o} \\
\text { s.t. } & P_{c h p}-x_{c h p, s}=P_{p r o} \\
& H_{c h p}+H_{\text {tes }}=H_{p r o}
\end{aligned}
$$

Where: $P_{s}$ is the price of electricity sold to the large grid; $P_{c h p}$ is the electricity generated by CHP; $H_{c h p}$ is the heat generated by CHP; $P_{\text {pro }}$ is the electricity sold by CHP operators to photovoltaic users; $H_{\text {pro }}$ is the thermal energy sold by CHP operators to photovoltaic users; $\gamma$ is the price of unit heat sold by the operator to the user; $\lambda$ is the price per unit of electricity sold by the operator to the user; $x_{c h p, s}$ is the electric energy sold by CHP to the large grid; $H_{\text {tes }}$ is the heat storage and heat release power, if it is the heat storage stage, then $H_{\text {tes }}=-H_{\text {in }}$, if it is the exothermic phase, then $H_{t e s}=H_{d r} ; C_{c h p}\left(P_{c h p}, H_{c h p}\right)$ is the cost function of CHP.

\subsection{User model}

\subsubsection{Electric load}

The user has a certain proportion of the load that can be translated, so it has a certain demand response capability. The user's electricity utility can be expressed as [9]:

$$
u_{n}=a_{n} x_{n}+b_{n}
$$

Where: $u_{n}$ is the electricity utility of user $\mathrm{n} ; x_{n}$ is the electricity consumption of user $\mathrm{n} ; a_{n}$ and $b_{n}$ are the electricity utility parameters respectively, and their values are adjusted according to the electricity consumption habits of users in each period. In the process of adjusting power consumption, it is necessary to ensure the normal power supply of the basic load, and also not exceed the upper limit of the power supply line, that is, $x_{n}^{\min } \leq x_{n} \leq x_{n}^{\max }$.

The output power of the user's photovoltaic system is affected by the capacity and the external environment (such as temperature, light, etc.). In the user's optimal scheduling process, the photovoltaic power $P_{P V, n}$ of the previous 24 hours needs to be predicted in advance. The role of the user depends on its net power $x_{n}-P_{P V, n}$.
The heating load of users is mainly supplied by CHP. From the literature [10], the temperature equation of state is:

$$
T_{t+1}^{i n}=T_{t}^{i n} e^{-1 / R C_{a i r}}+\left(R H_{t}^{a i r}+T_{t}^{o u t}\right)\left(1-e^{-1 / R C_{a i r}}\right)
$$

Where: $R$ is the thermal resistance of the building; $T_{t}^{\text {in }}$ is the indoor temperature at time $\mathrm{t} ; T_{t}^{\text {out }}$ is the outdoor temperature at time t; $C_{\text {air }}$ is the specific heat capacity of the air, which are all known quantities. Therefore, the indoor temperature and the input heat $H_{t}^{\text {air }}$ are approximately linear at time t.

\subsubsection{User Cost}

Considering the economy and the user's willingness to participate in demand response, the user's optimized operating cost includes the following parts: 1) transaction costs (or benefits) from large power grids; 2) uncomfortable costs due to flexible thermal load regulation; 3) Electricity utility; 4) the cost of purchasing electricity from CHP; 5) the cost of purchasing heat from CHP; 6) sharing the energy (or revenue) with other users. In the optimization operation, the user's role depends on its net load power, $C_{n}^{+}$is the cost of user $\mathrm{n}$ in the buyer role; $C_{n}^{-}$is the cost of user $\mathrm{n}$ in the seller role.

$$
\begin{aligned}
C_{n}^{+}= & P_{b}\left(x_{n}-P_{P V, n}-P_{p r o, n}-x_{n}^{\mathrm{in}}\right)+\alpha_{n}\left|Q_{T_{i n}^{n}}-Q_{T_{s e t}}\right| \\
& -\left(a_{n} x_{n}+b_{n}\right)+\gamma H_{p r o, n}+\lambda P_{p r o, n}+\xi x_{n}^{i n} \\
C_{n}^{-}= & -P_{s}\left(P_{P V, n}-x_{n}-x_{n}^{i n}\right)+\alpha_{n}\left|Q_{T_{i n}^{n}}+Q_{T_{s e t}}\right| \\
& -\left(a_{n} x_{n}+b_{n}\right)+\gamma H_{p r o, n}-\xi x_{n}^{i n}
\end{aligned}
$$

Where: $P_{b}$ is the price of electricity purchased from the large power grid; $P_{p r o, n}$ is the electricity sold by CHP to the users of $\mathrm{n} ; H_{p r o, n}$ is the heat energy sold by CHP to the users of $n ; \xi$ is the price of sharing electricity between users.

\subsection{Basic optimal scheduling model}

\subsubsection{Aiming at the best economy}

In the integrated energy system, CHP operators and PV users aim to minimize the operating cost of the system. The cost function $C_{c}$ is:

\subsubsection{Heating load}




$$
\begin{aligned}
\min C_{c}= & C_{\text {ope }}+\sum_{n=1}^{N} C_{n} \\
= & P_{b}\left(\sum_{n=1}^{N} x_{n}-\sum_{n=1}^{N} P_{P V, n}-P_{c h p}\right)^{+} \\
& -P_{s}\left(\sum_{n=1}^{N} P_{P V, n}+P_{c h p}-\sum_{n=1}^{N} x_{n}\right)^{+} \\
& +C_{c h p}\left(P_{c h p}, H_{c h p}\right)+\sum_{n=1}^{N} \alpha_{n}\left|Q_{T_{i n}^{n}}-Q_{T_{s a t}}\right| \\
& -\sum_{n=1}^{N}\left(a_{n} x_{n}+b_{n}\right)
\end{aligned}
$$

Where: $C_{n}$ is the operating cost of user $\mathrm{n}$; if user $\mathrm{n}$ is a buyer, then $C_{n}=C_{n}^{+}$; if it is a seller, then $C_{n}=C_{n}^{-} ;(x)^{+}=\max \{x, 0\}$.

\subsubsection{Aiming at maximizing user satisfaction}

\section{(1) Objective function}

The total operating cost of the system includes the operation and maintenance cost of each equipment and the fuel cost of the equipment in the system, namely:

$$
\min f_{1}=\sum_{t=1}^{24} \sum_{n=1}^{N}\left[M C_{n}(t)+P_{n}(t) \cdot \eta_{n}\right]
$$

Where $f_{1}$ is the total operating cost of the system; $N$ is the total number of equipment in the system, including fans, diesel engines, CHP units, gas boilers, batteries, etc.; $M C_{n}(t)$ is the maintenance cost of equipment $\mathrm{n}$ at time $\mathrm{t} ; P_{n}(t)$ is equipment $\mathrm{n}$ at time $\mathrm{t}$

The output power; $\eta_{n}$ is the cost required for the output unit power of the device $n$.

The user satisfaction level is:

$$
\max f_{2}=1-\frac{\sum_{t=1}^{24}\left(\left|P_{e}^{T L}(t)\right|+\left|P_{h}^{T L}(t)\right|\right)}{\sum_{t=1}^{24}\left[P_{e}(t)+P_{h}(t)\right]} \times 100 \%
$$

In the formula, $f_{2}$ is the user satisfaction level; $P_{e}^{T L}(t)$ and $P_{h}^{T L}(t)$ are the transferable electrical load and thermal load of the system respectively. Values greater than 0 indicate load transfer out, and less than 0 indicate load transfer in; $P_{e}(t)$ and $P_{h}(t)$ are the actual power and heat load demands of the system, respectively.

(2) Constraints

1) Energy balance constraints

$$
O=A I
$$

Where $O$ is the system energy output vector at time $\mathrm{t}$, that is, each load in the system; $A$ is the energy input and output conversion coefficient at time $\mathrm{t} ; I$ is the system energy input vector at time $t$.

Since the input primary energy is not a simple inputoutput relationship, there may be multiple transformation processes in a certain energy flow, so the relationship between the $i$-th energy and the $j$-th load conversion coefficient is:

$$
A_{i j}=\sum_{n=1}^{N}\left[V_{i, n}(t) \prod_{m=1}^{M} \eta_{n, m}^{j} \Phi(n, m, t)\right]
$$

Where $N$ is the total number of system equipment; $V_{i, n}(t)$ is the distribution coefficient of the primary energy $i$ allocated to the equipment $n ; M$ is the number of production links that the primary energy passes through; $\eta_{n, m}^{j}$ is the efficiency coefficient of the equipment $n$ being transformed into the $j$ th type of energy in the $m$ th link; $\Phi(n, m, t)$ is the $m$-th energy conversion relationship of device $n$ at time $t$.

2) CHP output constraints

$$
\begin{aligned}
& P_{n, t, \text { min }} \leq P_{n, t} \leq P_{n, t, \max } \quad n=1,2, \cdots, N \\
& \left\{\begin{array}{l}
P_{n, t-1}-P_{n, t} \leq P_{n, t}^{\text {down }} \\
P_{n, t}-P_{n, t-1} \leq P_{n, t}^{u p}
\end{array}\right.
\end{aligned}
$$

Where $P_{n, t, \min }$ is the minimum technical output of equipment $n$ at time $\mathrm{t} ; P_{n, t, \max }$ is the maximum technical output of equipment $n$ at time $\mathrm{t} ; P_{n, t}^{u p}$ and $P_{n, t}^{\text {down }}$ are the maximum values of the up and down ramp rates of equipment $n$, respectively.

3) Inequality constraints of energy storage system

During the charging and discharging process of the electric energy storage system, the system capacity constraints and charging and discharging speed constraints must be met. The specific model is as follows:

$$
\begin{aligned}
& S_{B E S}^{E, \min } \leq S_{B E S, t}^{E} \leq S_{B E S}^{E, \max } \\
& \left\{\begin{array}{l}
\delta_{c, t}^{E} P_{B E S, c}^{E, \min } \leq P_{B E S, c, t}^{E} \leq \delta_{c, t}^{E} P_{B E S, c}^{E, \max } \\
\delta_{d, t}^{E} P_{B E S, d}^{E, \min } \leq P_{B E S, d, t}^{E} \leq \delta_{d, t}^{E} P_{B E S, d}^{E, \max } \\
\delta_{c, t}^{E}+\delta_{d, t}^{E} \leq 1
\end{array}\right.
\end{aligned}
$$

In the formula, $S_{B E S}^{E, \min }$ and $S_{B E S}^{E, \max }$ are the minimum and maximum capacity of electric energy storage respectively; $\delta_{c, t}^{E}$ and $\delta_{d, t}^{E}$ are the charging and discharging state of electric energy storage at time $t$, and meet the different actions of charging and discharging; $P_{B E S, c}^{E, \text { min }}$ and $P_{B E S, c}^{E, \max }$ are the minimum and maximum charging rates respectively; $P_{B E S, d}^{E, \min }$ and $P_{B E S, d}^{E, \max }$ are the minimum and maximum Discharge rate.

The capacity constraints and charge-discharge rate constraints of thermal energy storage systems are similar to those of electrical energy storage systems.

(3) Multi-target processing

This paper adopts the method of min-max standardization to deal with the dimensional difference between the above two objective functions, and normalizes the objective function value of each problem. The expression is:

$$
f_{i}^{\prime}=\left(f_{i}-f_{\text {min }}\right) /\left(f_{i \max }-f_{i \min }\right), i=1,2
$$


In the formula, $f_{i}$ and $f^{\prime}$ are the actual value and normalized value of the objective function respectively; $f_{i \max }$ and $f_{i \min }$ are the maximum and minimum values obtained when only a single objective function is considered.

After the normalization is completed, the weighted sum method is used to transform the multi-objective optimization problem into a single-objective optimization problem, namely:

$$
\begin{gathered}
f=\omega_{1} f_{1}^{\prime}+\omega_{2} f_{2}^{\prime} \\
\omega_{1}+\omega_{2}=1
\end{gathered}
$$

In the formula, $\omega_{1}$ and $\omega_{2}$ are the weight coefficients corresponding to the objective function, which can be adjusted according to actual operating conditions.

\section{Research outlook}

With the rapid development of energy technology, the research on the optimization technology of integrated energy system operation based on multiple subjects is still a research hotspot. In the future, we can start from the following aspects:

(1) Optimization under uncertainty conditions. The optimal configuration of the multi-subject integrated energy system faces many uncertain factors, such as load changes, energy prices, and market environment. The existence of uncertain factors makes the future operating conditions of the integrated energy system may deviate from the design, and there are potential risks. Although some uncertainty sensitivity analysis has been carried out at present, there are few uncertain factors involved, simple problems, and no complete optimization system has been formed.

(2) Cooperative control technology. The highefficiency, economical and low-emission performance of the multi-subject integrated energy system under variable working conditions is inseparable from advanced control strategies and technologies. At present, the research on control thinking and operation strategy is relatively extensive, but the research on the regulation quality and collaborative control technology of the integrated energy system with multiple entities is still rare, and it cannot meet the control requirements of the co-supply system.

\section{Conclusion}

In this paper, for the integrated energy system including CHP operators and photovoltaic user groups, a demand response model that takes into account electrical and thermal energy is established. Through the configuration of thermal energy storage equipment, in order to minimize operating costs, CHP can be flexibly operated in a thermal setting. Electricity or electric heating mode; and established a multi-element-based integrated energy system distributed optimization scheduling model to improve the consumption rate of new energy and reduce operating costs; and for the shortcomings of existing research, proposed future research directions.

\section{Acknowledgments}

This paper is supported by "State Grid Ningxia Electric Power Co., Ltd. Management Technology Project (5229JY190013)".

\section{References}

1. Wang Chengshan, Lü Chaoxian, et al. Modeling and optimal operation of community integrated energy systems:a case study from China[J]. Applied energy,2018,230:1242-1254 .

2. Ren Hongbo, Wu Qiong, Ren Jianxing. Research on energy-saving benefits of natural gas distributed cogeneration system based on the demand side perspective[J]. Proceedings of the Chinese Society of Electrical Engineering, 2015, 35(17): 4430-4438.

3. Hu Landan, Liu Dong, Yan Lixia, etc. CCHP multienergy complementary optimization strategy considering demand response[J]. China Southern Power Grid Technology, 2016, 10(12): 75-81.

4. Gao Feng. Optimal design and coordinated control technology prospects of photovoltaic grid-connected systems[J]. China Southern Power Grid Technology, 2016, 10(3): 91-97.

5. Xue Jinhua, Ye Jilei, Tao Qiong, etc. Research on distributed photovoltaic operation strategies for different investment entities[J]. Power System Technology, 2017, 41(1): 93-98.

6. Chen Lei, Xu Fei, Wang Xiao, etc. Implementation method and effect analysis of heat storage to improve wind power absorption capacity[J]. Proceedings of the Chinese Society for Electrical Engineering, 2015, 35(17): 4283-4290.

7. MOHAMED F A, KOIVO H N. System modelling and online optimal management of microgrid using mesh adaptive direct search [J]. International Journal of Electrical Power \& Energy Systems ,2010,32(5):398 - 407.

8. CARRIóN M, etc. A computationally efficient mixed-integer linear formulation for the thermal unit commitment problem [J]. IEEE Transactions on Power Systems,2006,21(3):1371-1378 .

9. Wang Jie, Liu Nian. Distributed optimization operation method of multi-agent integrated energy system[J].Southern Power Grid Technology,2018,12(03),98-104.

10. BRAHMAN F, etc.Optimal electrical and thermal energy management of a residential energy hub, integrating demand response and energy storage system [J].Energy \& Buildings, 2015(90) :65-75. 